\title{
STRUCTURE AND SPECTROSCOPIC BEHAVIOUR OF ADDUCT OF 2,6-DICHLORO-4-NITROPHENOL WITH 2,4,6-TRIMETHYLPYRIDIINE
}

\author{
I. Majerz, W. SaWka-Dobrowolska and L. SobczyK \\ Institute of Chemistry, University of Wroclaw \\ F. Joliot-Curie 14, 50-383 Wrocław, Poland
}

(Received April 7, 1995)

\begin{abstract}
Dedicated to Professors Krzysztof Pigoń, Józef W. Rohleder and Zdzisław Ruziewicz on the occasion of their 70th birthdays*
\end{abstract}

2,4,6-trimethylpyridinium 2,6-dichloro-4-nitrophenolate crystallizes in monoclinic system, space group $P 2_{1} / c, a=7.534(2), b=26.673(5), c=$ 8.452(2) $\AA, \beta=116.16(3)^{\circ}, Z=4$. The planar molecules are arranged in the lattice as antiparallel oriented pairs with the symmetry centre. The $\mathrm{O}^{-} \ldots \mathrm{H}-\mathrm{N}^{+}$hydrogen bonds are relatively long, 2.686(4) $\AA$. The $\mathrm{C}-\mathrm{O}$ bond length, $1.250(4) \AA$, indicates an extended charge separation. The IR and UV-VIS spectra in $\mathrm{KBr}$ pellets confirm the ion pairs to be present in the solid state.

PACS numbers: $61.10 . \mathrm{My}, 33.20 . \mathrm{Ea}, 33.20 . \mathrm{Lg}$

\section{Introduction}

The studies on the structure and spectroscopic properties of adducts of pentachlorophenol (PCP) and 2,6-dichloro-4-nitrophenol (DCNP) with amines, performed so far [1], showed that at suitable choice of acidity and basicity of interacting components the hydrogen bonds $\mathrm{O}-\mathrm{H} \cdots \mathrm{N}$ are formed in which the proton is shared between the bridge atoms. Such bonds are very short, their length oscillates around $2.5 \AA$. In the IR spectra vanishing of absorption above $1700 \mathrm{~cm}^{-1}$ takes place and in the fingerprint region one observes a broad absorption assigned to the protonic vibrations. There is a close analogy to the complexes of carboxylic acids with amines [2]. The problem of the potential for the proton motion is still open.

*Submitted on invitation of the Institute of Physical and Theoretical Chemistry, Technical University of Wrocław, Wrocław, Poland. 
In the case of weak bases the bridge hydrogen atom is localized at the oxygen atom, while for strong bases the hydrogen atom is attached to the nitrogen atom with creation of the $\mathrm{O}^{-} \ldots \mathrm{H}-\mathrm{N}^{+}$ion pair. The charge transfer is well reflected in the length of the phenolic $\mathrm{C}-\mathrm{O}$ bond [3].

In the present paper we would like to present the results of the studies on the structure and spectroscopic behaviour of adduct of DCNP with 2,4,6-trimethylpyridine (TMP). The considerable basicity of this compound should favour the creation of the ion pair. On the other hand, one could expect the influence of the steric effect on the geometry of the hydrogen bond and the structure of the adduct. The presence of methyl groups and chlorine atoms in the ortho position should counteract to the coplanar arrangement of the phenyl rings.

\section{Experimental}

The complex was obtained from equimolar amount of TMP and DCNP in an acetonitrile solution. The yellow crystals were grown from $\mathrm{CCl}_{4}$ by slow isothermal evaporation of the solvent.

All X-ray measurements were made on a KUMA KM-4 (Kuma Diffraction, Poland) computer-controlled $\kappa$-axis four-circle diffractometer with graphite monochromated $\mathrm{Cu} K_{\alpha}$ radiation at room temperature. The stability of crystal was monitored by measurements of three standards every 100 reflections. The data were corrected for Lorentz and polarization effects as well as for absorption [4] (correction between 0.981 and 1.261). Experimental details are given in Table I.

The crystal structure was solved by direct methods with SHELXS-86 [5] and refined by full-matrix least squares technique using SHELXL-93 [6] with anisotropic thermal parameters for non- $\mathrm{H}$ atoms. At intermediate stages of the refinement, the difference maps showed all $\mathrm{H}$ atoms. In the final cycles of the refinement $\mathrm{H}$-atom parameters with isotropic thermal parameters were included. Scattering factors were those incorporated in SHELXL-93.

IR spectrum was recorded for pellets in $\mathrm{KBr}$ on a Bruker FTIR IFS $113 \mathrm{v}$ spectrophotometer. UV spectra of solid compounds were recorded on Cary 1 UV-VIS spectrometer in $\mathrm{KBr}$ pellets.

\section{Results and discussion}

The scheme of the adduct with numbering of atoms is presented in Fig. 1. The atom coordinates are listed in Tables II and IV, while the bond lengths and angles are contained in Table V. Table III contains anisotropic thermal parameters. The arrangement of adducts in the lattice are depicted in Fig. 2.

Unexpectedly it appeared that the phenyl and pyridinium rings in the adduct are coplanar in spite of a steric hindrance. The angle between normals to these planes is $2.9(1)^{\circ}$. The $\mathrm{O}^{-} \cdots \mathrm{H}-\mathrm{N}^{+}$hydrogen bond is $2.686(4) \AA$ long. The $\mathrm{O}(1) \mathrm{H}(1) \mathrm{N}(1)$ angle is $173(4)^{\circ}$, so that the axis along the adduct is close to the twofold screw symmetry axis. It is parallel to the $b$ axis. The $\mathrm{C}(1)-\mathrm{O}(1)$ bond is very short, the shortest among the $\mathrm{C}-\mathrm{O}$ bonds known so far in adducts of phenols with amines $[1,7]$. It means a notable charge separation. 
TABLE I

Summary of crystal data, data collection, and refinement conditions.

\begin{tabular}{|c|c|}
\hline Compound & $\mathrm{C}_{6} \mathrm{H}_{2} \mathrm{Cl}_{2} \mathrm{NO}_{2} \mathrm{O}^{-} \cdot \mathrm{C}_{8} \mathrm{H}_{11} \mathrm{NH}^{+}$ \\
\hline Molecular weight & 329.17 \\
\hline Space group & $P 2_{1} / c$ (No. 14 ) \\
\hline Crystal system & monoclinic \\
\hline Temperature $[\mathrm{K}]$ & $293 \pm 2$ \\
\hline Cell constants & $\left(25\right.$ reflections $\left.22<2 \Theta<34^{\circ}\right)$ \\
\hline$a[\AA]$ & $7.534(2)$ \\
\hline$b[\AA]$ & $26.673(5)$ \\
\hline$c[\AA]$ & $8.452(2)$ \\
\hline$\beta[\operatorname{deg}]$ & $116.16(3)$ \\
\hline Cell volume $\left[\AA^{3}\right]$ & $1524.5(6)$ \\
\hline Formula units/unit cell & 4 \\
\hline$D_{\text {calc }}\left[\mathrm{g} \mathrm{cm}^{-3}\right]$ & $1.434(1)$ \\
\hline $\mathrm{D}_{\mathrm{m}}\left[\mathrm{g} \mathrm{cm}^{-3}\right]^{*}$ & $1.43(1)$ \\
\hline$\mu\left(\mathrm{Cu} K_{\alpha}\right)\left[\mathrm{mm}^{-1}\right]$ & 3.94 \\
\hline Diffractometer/scan & KUMA KM $4, \omega / 2 \Theta$ \\
\hline Radiation & $\mathrm{Cu} K_{\alpha}, \lambda=1.5418 \AA$ \\
\hline Max. crystal dimensions [mm] & $0.3 \times 0.2 \times 0.5$ \\
\hline Standard reflections & 3 (100 ref) \\
\hline Decay of standards $[\%]$ & 2 \\
\hline Reflections measured & 2928 \\
\hline $2 \Theta$ range $[\mathrm{deg}]$ & $6.0-140^{\circ}$ \\
\hline Ranges of $h, k, l$ & $0-8 ; 0-25 ;-10-9$ \\
\hline Reflections observed $\left[F_{0} \geq 5 \sigma(F)\right]$ & 2000 \\
\hline Corrections applied & $\begin{array}{l}\text { Lorentz; polarization; } \\
\text { absorption }\end{array}$ \\
\hline Computer programs & SHELXS-86; SHELXL-93 \\
\hline Source of structure factors used & programs \\
\hline Structure solution & direct methods \\
\hline Treatment of hydrogen atoms & isotropic \\
\hline No. of parameters varied & 246 \\
\hline Weights** $^{*}(a, b, f)$ & $0.0873 ; 0.9816 ; 1 / 3$ \\
\hline GOF & 1.071 \\
\hline$R_{1}=\sum\left(\left|F_{0}\right|-\left|F_{c}\right|\right) /\left|F_{0}\right|$ & 0.0479 \\
\hline$w R 2=\left\{\sum\left[w\left(F_{0}^{2}-F_{c}^{2}\right)^{2}\right] / \sum\left[w\left(F_{c}^{2}\right)^{2}\right]\right\}^{1 / 2 * *}$ & 0.1382 \\
\hline Largest feature final diff. map & $0.35 /-0.274$ \\
\hline
\end{tabular}

${ }^{*}$ Measured by flotation in $\mathrm{CCl}_{4}$ /ethyl bromide.

${ }^{* *} w=1 /\left[\sigma^{2}\left(F_{0}^{2}\right)+(a P)^{2}+b P\right]$ where $P=\left[f \cdot \operatorname{Max}\right.$ of $\left(0\right.$ or $\left.\left.\left.F_{0}^{2}\right)+(1-f) F_{c}^{2}\right)\right]$. 


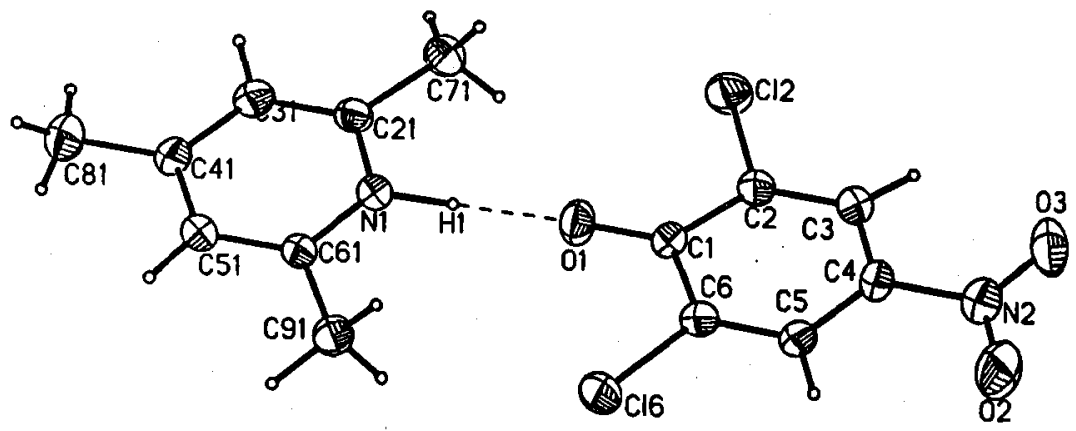

Fig. 1. Molecular structure of TMP DCNP complex and numbering of atoms. Displacement ellipsoids of non-H atoms are at the $30 \%$ probability level.

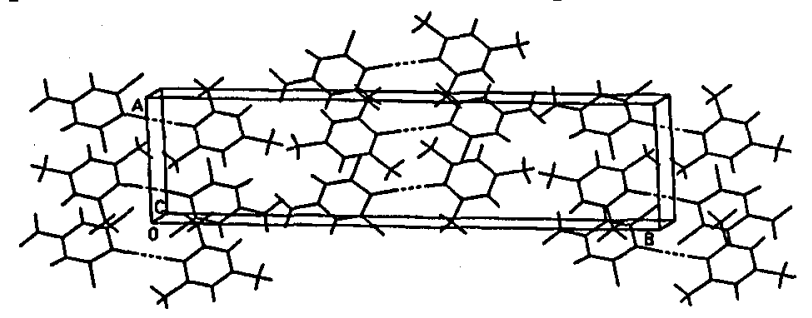

Fig. 2. Arrangement of TMP DCNP in the lattice.

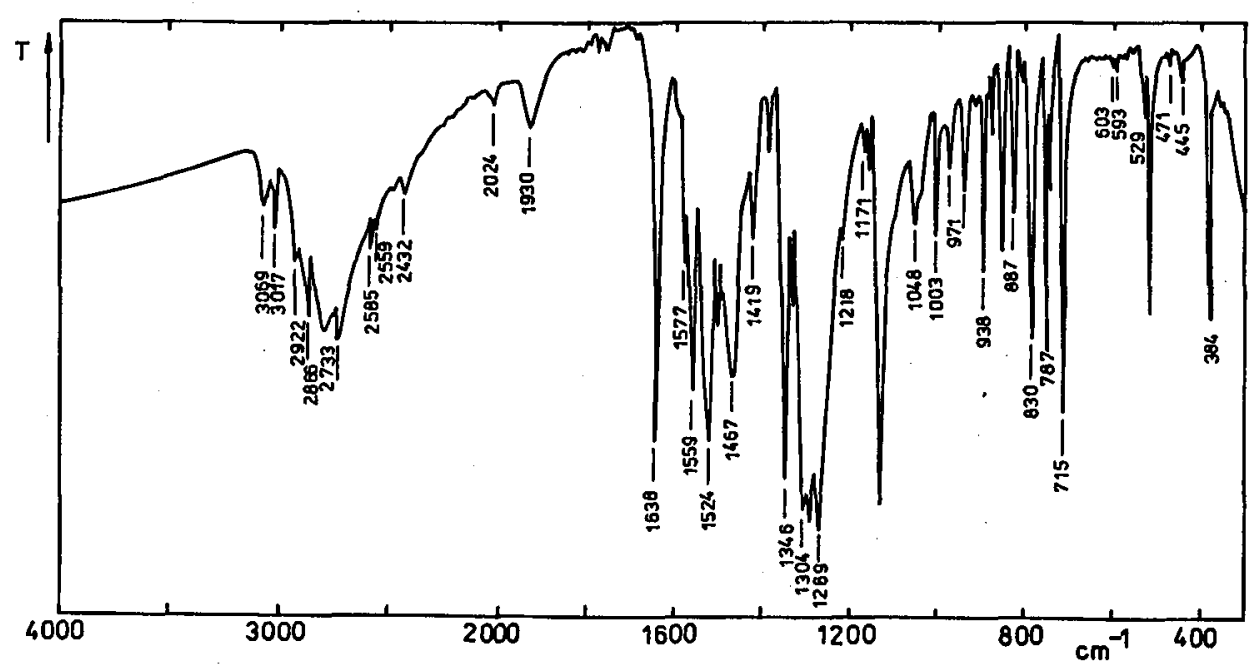

Fig. 3. Infrared spectrum (In KBr disc) of TMP DCNP.

The H-bond complexes form in the lattice the pairs with an opposite orientation of dipoles and paralel arrangement of the phenyl rings. Thus, the pairs of adducts possess the symmetry centre. The distance between the parallel phenyl 
and pyridine atoms is within the $3.44(3)-3.57(4) \AA$ range. It is close to the van der Waals contacts between the aromatic rings $(3.6 \AA)$. The coplanarity of the phenyl and pyridine rings and the parallel arrangement in pairs is stabilized by the dipole-dipole interaction and, in addition, by the sandwich type charge transfer interaction between the 2,6-dichloro-4-nitrophenolic (electron donor) and trimethylpyridyl (electron acceptor) groupings. A quinoid character of phenolic ring is expressed to some extent. Thus the $\mathrm{C}(2)-\mathrm{C}(3)$ and $\mathrm{C}(5)-\mathrm{C}(6)$ bond lengths are equal to $1.347(5)$ and $1.350(5) \AA$ respectively. Simultaneously, a shortening of the $\mathrm{N}(2)-\mathrm{C}(4)$ bond length is observed (1.425(5) $\AA$ ). As far as the contact between pairs is concerned one can distinguish interactions between the oxygen atoms of

TABLE II

Atomic coordinates $\left(\times 10^{4}\right)\left(\times 10^{5}\right.$ for $\mathrm{Cl}$ atoms $)$ and equivalent isotropic displacement parameters $\left(\AA^{2} \times 10^{4}\right)$ for 2,4,6-trimethylpyridinium 2,6-dichloro-4-nitrophenolate. $U_{\mathrm{eq}}$ is defined as one third of the trace of the orthogonalized $U_{i j}$ tensor.

\begin{tabular}{l|c|c|c|c}
\hline \hline & $x$ & $y$ & $z$ & $U_{\text {eq }}$ \\
\hline $\mathrm{Cl}(2)$ & $-49666(14)$ & $60773(4)$ & $23713(10)$ & $677(3)$ \\
$\mathrm{Cl}(6)$ & $7896(13)$ & $54709(3)$ & $86233(10)$ & $604(3)$ \\
$\mathrm{O}(1)$ & $-244(4)$ & $5351(1)$ & $5045(3)$ & $613(7)$ \\
$\mathrm{O}(2)$ & $250(6)$ & $7399(1)$ & $8759(5)$ & $1144(13)$ \\
$\mathrm{O}(3)$ & $-2087(6)$ & $7644(1)$ & $6336(6)$ & $1080(12)$ \\
$\mathrm{N}(1)$ & $-2983(4)$ & $4363(1)$ & $4402(3)$ & $440(6)$ \\
$\mathrm{N}(2)$ & $-1063(6)$ & $7314(1)$ & $7293(5)$ & $771(10)$ \\
$\mathrm{C}(1)$ & $-2122(5)$ & $5798(1)$ & $5543(4)$ & $446(7)$ \\
$\mathrm{C}(2)$ & $-3156(5)$ & $6217(1)$ & $4483(4)$ & $481(7)$ \\
$\mathrm{C}(3)$ & $-2834(5)$ & $6698(1)$ & $5010(5)$ & $549(8)$ \\
$\mathrm{C}(4)$ & $-1401(5)$ & $6807(1)$ & $6699(5)$ & $558(8)$ \\
$\mathrm{C}(5)$ & $-289(5)$ & $6426(1)$ & $7821(4)$ & $503(8)$ \\
$\mathrm{C}(6)$ & $-631(5)$ & $5947(1)$ & $7250(4)$ & $467(7)$ \\
$\mathrm{C}(21)$ & $-4462(4)$ & $4255(1)$ & $2799(4)$ & $463(7)$ \\
$\mathrm{C}(31)$ & $-4774(5)$ & $3771(1)$ & $2262(4)$ & $524(8)$ \\
$\mathrm{C}(41)$ & $-3634(5)$ & $3384(1)$ & $3334(4)$ & $530(8)$ \\
$\mathrm{C}(51)^{\prime}$ & $-2154(5)$ & $3516(1)$ & $4969(5)$ & $522(8)$ \\
$\mathrm{C}(61)$ & $-1828(4)$ & $4001(1)$ & $5503(4)$ & $436(7)$ \\
$\mathrm{C}(71)$ & $-5648(7)$ & $4694(2)$ & $1732(5)$ & $619(10)$ \\
$\mathrm{C}(81)$ & $-3975(9)$ & $2857(2)$ & $2746(8)$ & $831(14)$ \\
$\mathrm{C}(91)$ & $-245(6)$ & $4169(2)$ & $7237(5)$ & $577(9)$ \\
\hline$U_{\text {eq }}=(1 / 3) \sum_{i} \sum_{j} U_{i j} a_{i}^{*} a_{j}^{*} a_{i} a_{j}$. &
\end{tabular}


nitro groups and hydrogen atoms of $\mathrm{C}_{\mathrm{ar}}-\mathrm{H}$ groups of the trimethylpyridinium ring. The $\mathrm{C}(51)-\mathrm{H}(51) \cdots \mathrm{O}(2)(-x,-0.5+y,-z+1)$ distance equals $3.274(5) \AA$ with the $\mathrm{C}(51)-\mathrm{H}(51) \cdots \mathrm{O}(2)(-x,-0.5+y,-z+1)$ angle equal to $\AA 148(4)^{\circ}$ and the $\mathrm{H}(51) \ldots$ $\mathrm{O}(2)$ distance is $2.44(4) \AA$.

The ionic feature of the OHN hydrogen bridge is well reflected in IR and UV-VIS spectra. The IR spectrum is shown in Fig. 3. The stretching vibration band is located at particularly high frequencies, the highest of which has been observed for amine phenolates. The gravity centre of this band lies at about $2860 \mathrm{~cm}^{-1}$. The characteristic feature of the $\nu\left(\mathrm{NH}^{+}\right)$band is a rich fine structure typical of bands located in the $2500-3000 \mathrm{~cm}^{-1}$ range.

TABLE III Anisotropic thermal parameters of 2,4,6-trimethylpyridinium 2,6-dichloro-4-nitrophe nolate with e.s.d. values in parentheses.

\begin{tabular}{l|r|r|r|r|r|r}
\hline \hline Atom & $U_{11}$ & $U_{22}$ & $U_{33}$ & $U_{12}$ & $U_{13}$ & $U_{23}$ \\
\hline $\mathrm{Cl}(2)$ & $58(1)$ & $76(1)$ & $50(1)$ & $-1(1)$ & $7(1)$ & $3(1)$ \\
$\mathrm{Cl}(6)$ & $56(1)$ & $60(1)$ & $55(1)$ & $10(1)$ & $15(1)$ & $9(1)$ \\
$\mathrm{O}(1)$ & $71(2)$ & $42(2)$ & $61(1)$ & $-6(1)$ & $20(1)$ & $-4(1)$ \\
$\mathrm{O}(3)$ & $115(3)$ & $47(2)$ & $134(3)$ & $-1(2)$ & $29(2)$ & $7(2)$ \\
$\mathrm{O}(2)$ & $112(3)$ & $71(2)$ & $116(3)$ & $-39(2)$ & $10(2)$ & $-12(2)$ \\
$\mathrm{N}(1)$ & $42(2)$ & $42(2)$ & $47(1)$ & $0(1)$ & $19(1)$ & $1(1)$ \\
$\mathrm{N}(2)$ & $68(2)$ & $55(2)$ & $100(3)$ & $-15(2)$ & $29(2)$ & $-7(2)$ \\
$\mathrm{C}(1)$ & $42(2)$ & $42(2)$ & $49(2)$ & $-1(1)$ & $20(1)$ & $-3(1)$ \\
$\mathrm{C}(2)$ & $45(2)$ & $48(2)$ & $47(2)$ & $0(1)$ & $16(1)$ & $1(1)$ \\
$\mathrm{C}(3)$ & $48(2)$ & $45(2)$ & $64(2)$ & $6(2)$ & $19(2)$ & $2(2)$ \\
$\mathrm{C}(4)$ & $53(2)$ & $38(2)$ & $75(2)$ & $-7(2)$ & $27(2)$ & $-5(2)$ \\
$\mathrm{C}(5)$ & $43(2)$ & $48(2)$ & $55(2)$ & $-7(1)$ & $17(1)$ & $-4(1)$ \\
$\mathrm{C}(6)$ & $41(2)$ & $51(2)$ & $46(1)$ & $1(1)$ & $17(1)$ & $-1(1)$ \\
$\mathrm{C}(21)$ & $39(2)$ & $51(2)$ & $46(1)$ & $7(1)$ & $16(1)$ & $2(1)$ \\
$\mathrm{C}(31)$ & $46(2)$ & $58(2)$ & $48(2)$ & $-2(1)$ & $16(1)$ & $-5(2)$ \\
$\mathrm{C}(41)$ & $50(2)$ & $48(2)$ & $58(2)$ & $-8(1)$ & $20(1)$ & $-4(2)$ \\
$\mathrm{C}(51)$ & $47(2)$ & $46(2)$ & $59(2)$ & $3(1)$ & $19(1)$ & $5(2)$ \\
$\mathrm{C}(61)$ & $41(2)$ & $42(2)$ & $44(1)$ & $1(1)$ & $15(1)$ & $2(1)$ \\
$\mathrm{C}(71)$ & $57(2)$ & $57(2)$ & $61(2)$ & $10(2)$ & $17(2)$ & $2(2)$ \\
$\mathrm{C}(81)$ & $79(4)$ & $53(3)$ & $97(3)$ & $-18(2)$ & $20(3)$ & $-6(2)$ \\
$\mathrm{C}(91)$ & $53(2)$ & $56(2)$ & $52(2)$ & $-5(2)$ & $12(2)$ & $-1(2)$ \\
\hline$U$ & $\ln \left(-2 \pi\left(h^{2} a^{* 2} U_{11}+k^{2} b^{* 2} U_{22}\right.\right.$ & $+l^{2} c^{* 2} U_{33}+$ \\
$\left.\left.2 h k a^{*} b^{*} U_{12}+2 h l a^{*} c^{*} U_{13}+2 k l b^{*} c^{*} U_{23}\right)\right]$ & & \\
& \multicolumn{6}{|l}{}
\end{tabular}


TABLE IV

Hydrogen coordinates $\left(\times 10^{4}\right)$ and isotropic displacement parameters $\left(\AA^{2} \times 10^{3}\right)$ for 2,4,6-trimethylpyridinium 2,6-dichloro-4-nitrophenolate.

\begin{tabular}{l|c|c|c|c}
\hline \hline & $x$ & $y$ & $z$ & $U_{\text {eq }}$ \\
\hline $\mathrm{H}(1)$ & $-2788(60)$ & $4741(17)$ & $4739(53)$ & $76(12)$ \\
$\mathrm{H}(3)$ & $-3440(61)$ & $6980(17)$ & $4253(52)$ & $74(12)$ \\
$\mathrm{H}(5)$ & $744(62)$ & $6507(15)$ & $8926(54)$ & $72(12)$ \\
$\mathrm{H}(51)$ & $-1295(63)$ & $3273(16)$ & $5747(53)$ & $72(11)$ \\
$\mathrm{H}(31)$ & $--5868(62)$ & $3698(16)$ & $1192(54)$ & $70(12)$ \\
$\mathrm{H}(121)$ & $-4875(73)$ & $4956(20)$ & $1864(60)$ & $86(15)$ \\
$\mathrm{H}(122)$ & $-6204(86)$ & $4860(22)$ & $2379(75)$ & $115(19)$ \\
$\mathrm{H}(123)$ & $-6380(111)$ & $4619(26)$ & $538(100)$ & $150(25)$ \\
$\mathrm{H}(131)$ & $-2960(122)$ & $2749(29)$ & $2617(94)$ & $117(30)$ \\
$\mathrm{H}(132)$ & $-4904(137)$ & $2881(34)$ & $1634(117)$ & $112(33)$ \\
$\mathrm{H}(133)$ & $-4255(131)$ & $2625(36)$ & $3470(113)$ & $124(36)$ \\
$\mathrm{H}(181)$ & $680(112)$ & $3875(28)$ & $7924(95)$ & $105(26)$ \\
$\mathrm{H}(182)$ & $-836(78)$ & $4373(21)$ & $7915(67)$ & $106(17)$ \\
$\mathrm{H}(183)$ & $548(73)$ & $4405(20)$ & $7106(62)$ & $89(15)$
\end{tabular}

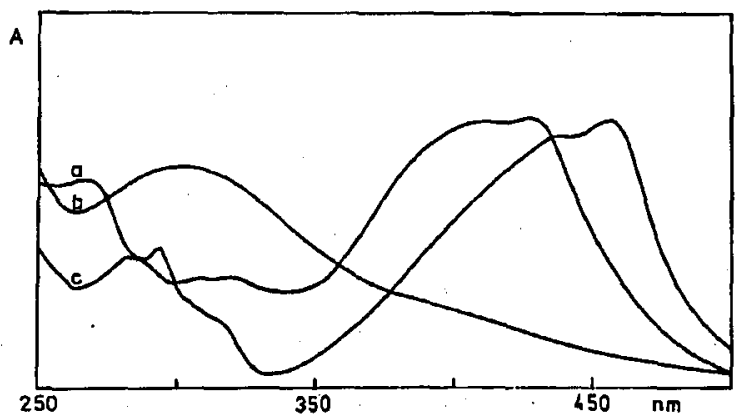

Fig. 4. UV-VIS spectra in KBr disc: $a-$ TMP DCNP, $b-$ DCNP, $c-$ tetrabutyl/ammonium salt of DCNP.

Figure 4 shows the UV-VIS spectra of DCNP itself and TMP DCNP adduct and tetramethylammonium salt in the solid state ( $\mathrm{KBr}$ pellets). The absorption band of the adduct is located at about $415 \mathrm{~nm}$, while of the tetrabutylammonium salt at about $445 \mathrm{~nm}$. If we compare these data with the spectrum of TMP DCNP in dichloroethane [8] (the band localized at $400 \mathrm{~nm}$ ) we can conclude that the solid state promotes a larger charge separation than in solution. This is in agreement with the IR spectra and packing in the solid state. The antiparallel arrangement 
of the hydrogen bonded complexes in a pair favours the charge separation. Nevertheless, such a charge separation is much smaller than in the tetrabutylammonium salt.

TABLE V

Bond lengths $(\AA)$ and bond angles (deg) with e.s.d. values in parentheses for the complex of 2,4,6-trimethylpyridine with 2,6-dichloro-4-nitrophenol.

\begin{tabular}{l|r||l|l}
\hline \hline \multicolumn{2}{c||}{ Bond lengths } & \multicolumn{2}{|c}{ Bond angles } \\
\hline $\mathrm{N}(1)^{+} \ldots \mathrm{O}(1)^{-}$ & $2.686(4)$ & $\mathrm{N}(1)^{+}-\mathrm{H}(1) \cdots \mathrm{O}(1)^{-}$ & $173(4)$ \\
$\mathrm{N}(1)-\mathrm{H}(1)$ & $1.04(5)$ & $\mathrm{C}(21)-\mathrm{N}(1)-\mathrm{C}(61)$ & $122.2(3)$ \\
$\mathrm{O}(1) \cdots \mathrm{H}(1)$ & $1.65(5)$ & $\mathrm{O}(3)-\mathrm{N}(2)-\mathrm{O}(2)$ & $122.1(4)$ \\
$\mathrm{O}(1)-\mathrm{C}(1)$ & $1.250(4)$ & $\mathrm{O}(3)-\mathrm{N}(2)-\mathrm{C}(4)$ & $119.5(4)$ \\
$\mathrm{Cl}(2)-\mathrm{C}(2)$ & $1.743(3)$ & $\mathrm{O}(2)-\mathrm{N}(2)-\mathrm{C}(4)$ & $118.4(4)$ \\
$\mathrm{Cl}(6)-\mathrm{C}(6)$ & $1.735(3)$ & $\mathrm{O}(1)-\mathrm{C}(1)-\mathrm{C}(2)$ & $124.3(3)$ \\
$\mathrm{O}(3)-\mathrm{N}(2)$ & $1.216(5)$ & $\mathrm{O}(1)-\mathrm{C}(1)-\mathrm{C}(6)$ & $123.3(3)$ \\
$\mathrm{O}(2)-\mathrm{N}(2)$ & $1.219(5)$ & $\mathrm{C}(2)-\mathrm{C}(1)-\mathrm{C}(6)$ & $112.4(3)$ \\
$\mathrm{N}(1)-\mathrm{C}(21)$ & $1.352(4)$ & $\mathrm{C}(3)-\mathrm{C}(2)-\mathrm{C}(1)$ & $124.7(3)$ \\
$\mathrm{N}(1)-\mathrm{C}(61)$ & $1.356(4)$ & $\mathrm{C}(3)-\mathrm{C}(2)-\mathrm{Cl}(2)$ & $119.2(3)$ \\
$\mathrm{N}(2)-\mathrm{C}(4)$ & $1.425(5)$ & $\mathrm{C}(1)-\mathrm{C}(2)-\mathrm{Cl}(2)$ & $116.1(2)$ \\
$\mathrm{C}(1)-\mathrm{C}(2)$ & $1.429(5)$ & $\mathrm{C}(2)-\mathrm{C}(3)-\mathrm{C}(4)$ & $119.0(3)$ \\
$\mathrm{C}(1)-\mathrm{C}(6)$ & $1.440(4)$ & $\mathrm{C}(3)-\mathrm{C}(4)-\mathrm{C}(5)$ & $120.7(3)$ \\
$\mathrm{C}(2)-\mathrm{C}(3)$ & $1.347(5)$ & $\mathrm{C}(3)-\mathrm{C}(4)-\mathrm{N}(2)$ & $119.8(4)$ \\
$\mathrm{C}(3)-\mathrm{C}(4)$ & $1.388(5)$ & $\mathrm{C}(5)-\mathrm{C}(4)-\mathrm{N}(2)$ & $119.5(3)$ \\
$\mathrm{C}(4)-\mathrm{C}(5)$ & $1.391(5)$ & $\mathrm{C}(6)-\mathrm{C}(5)-\mathrm{C}(4)$ & $119.0(3)$ \\
$\mathrm{C}(5)-\mathrm{C}(6)$ & $1.350(5)$ & $\mathrm{C}(5)-\mathrm{C}(6)-\mathrm{C}(1)$ & $124.2(3)$ \\
$\mathrm{C}(21)-\mathrm{C}(31)$ & $1.355(5)$ & $\mathrm{C}(5)-\mathrm{C}(6)-\mathrm{Cl}(6)$ & $119.3(2)$ \\
$\mathrm{C}(31)-\mathrm{C}(41)$ & $1.393(5)$ & $\mathrm{C}(1)-\mathrm{C}(6)-\mathrm{Cl}(6)$ & $116.5(2)$ \\
$\mathrm{C}(41)-\mathrm{C}(51)$ & $1.386(5)$ & $\mathrm{N}(1)-\mathrm{C}(21)-\mathrm{C}(31)$ & $118.9(3)$ \\
$\mathrm{C}(51)-\mathrm{C}(61)$ & $1.358(5)$ & $\mathrm{N}(1)-\mathrm{C}(21)-\mathrm{C}(71)$ & $116.4(3)$ \\
$\mathrm{C}(41)-\mathrm{C}(81)$ & $1.473(6)$ & $\mathrm{C}(31)-\mathrm{C}(21)-\mathrm{C}(71)$ & $124.7(3)$ \\
$\mathrm{C}(21)-\mathrm{C}(71)$ & $1.506(5)$ & $\mathrm{C}(21)-\mathrm{C}(31)-\mathrm{C}(41)$ & $121.6(3)$ \\
$\mathrm{C}(61)-\mathrm{C}(91)$ & $1.493(4)$ & $\mathrm{C}(51)-\mathrm{C}(41)-\mathrm{C}(31)$ & $117.0(3)$ \\
$\mathrm{C}-\mathrm{II}$ & $0.87(8)-1.04(8)$ & $\mathrm{C}(51)-\mathrm{C}(41)-\mathrm{C}(81)$ & $121.5(4)$ \\
& & $\mathrm{C}(31)-\mathrm{C}(41)-\mathrm{C}(81)$ & $121.5(4)$ \\
& & $\mathrm{C}(61)-\mathrm{C}(51)-\mathrm{C}(41)$ & $121.5(3)$ \\
& & $\mathrm{N}(1)-\mathrm{C}(61)-\mathrm{C}(51)$ & $118.9(3)$ \\
& & $\mathrm{N}(1)-\mathrm{C}(61)-\mathrm{C}(91)$ & $117.0(3)$ \\
& & $\mathrm{C}(51)-\mathrm{C}(61)-\mathrm{C}(91)$ & $124.1(3)$ \\
& & & \\
& & & \\
& & & \\
& & &
\end{tabular}




\section{References}

[1] I. Majerz, W. Sawka-Dobrowolska, L. Sobczyk, Pol. J. Chem. 67, 1657 (1993); I. Majerz, W. Sawka-Dobrowolska, L. Sobczyk, J. Mol. Struct. 297, 177 (1993); I. Majerz, W. Sawka-Dobrowolska, L. Sobczyk, J. Mol. Struct. 319, 1 (1994); I. Majerz, W. Sawka-Dobrowolska, submitted to J. Chem. Cryst.

[2] S.L. Johnson, K.A. Rumon, J. Phys. Chem. 69, 74 (1965).

[3] I. Majerz, Z. Malarski, L. Sobczyk, in preparation.

[4] F. Uguzzoli, Comput. Chem. 411, 109 (1987).

[5] G.M. Sheldrick, Acta Crystallogr. A 46, 467 (1990).

[6] G.M. Sheldrick, SHELXL93, Program for the Refinement of Crystal Structures, Univ. of Göttingen, Germany (1994).

[7] I. Majerz, Z. Malarski, W. Sawka-Dobrowolska, J. Mol. Struct. 249, 109 (1991) and references cited therein.

[8] H. Romanowski, L. Sobczyk, J. Phys. Chem. 79, 2535 (1975). 